\title{
A Case of A Mesenteric Cyst Mimicking a Biloma
}

\author{
Mohamad Fekredeen Ayas ${ }^{\mathrm{a}, \mathrm{e}}$, Saif Affas ${ }^{\mathrm{a}}$, Gilles Jadd Hoilat ${ }^{\mathrm{b}}$, \\ Ihab Kassab ${ }^{\mathrm{c}}$, Mohammed Barawi ${ }^{\mathrm{d}}$
}

\begin{abstract}
Mesenteric cysts are a rare entity, with approximately 1,000 cases reported in the literature. Its etiology is unknown but was theorized to be a benign ectopic lymphatic proliferation in the mesentery. Imaging with surgical excision and pathologic microscopic evaluation is needed for diagnosis, but no specific guidelines for its management have been documented. Per most cases in the literature, drainage of mesenteric cysts is suboptimal and associated with increased risk of recurrence and infection, making surgical resection the treatment of choice. With its varying locations and presentations, diagnosis can be tricky especially given the rarity of its occurrence. Differential diagnosis includes pancreatic pseudocyst, hemangioma, choledochal cyst, hydatid cyst, cystic teratoma, etc. We present a 35-year-old woman who came in with worsening right upper quadrant (RUQ) pain due to chronic cholelithiasis and was found to have a large RUQ cystic mass adherent to the gallbladder, suggesting possible biloma or pancreatic pseudocyst; however, tissue analysis was positive for a mesenteric cyst.
\end{abstract}

Keywords: Mesenteric cyst; Biloma; Surgical resection

\section{Introduction}

Mesenteric cysts are rare benign intra-abdominal tumors with an incidence of approximately 1 case per 100,000 - 250,000 hospital admissions [1]. They are defined as any cyst in the mesentery of the gastrointestinal tract, most commonly found in the small bowel mesentery (ileum: 60\%), followed by large

Manuscript submitted October 17, 2020, accepted November 24, 2020

Published online December 23, 2020

aDepartment of Internal Medicine, Ascension St. John Hospital, Detroit, MI, USA

${ }^{b}$ Department of Internal Medicine, SUNY Upstate Medical University, Syracuse, NY, USA

${ }^{\mathrm{c}}$ Division of Hospital Medicine, Department of Internal Medicine, University of Michigan, Ann Arbor, MI, USA

dDivision of Gastroenterology and Hepatology, Ascension St. John Hospital, Detroit, MI, USA

e Corresponding Author: Mohamad Fekredeen Ayas, Department of Internal Medicine, Ascension St. John Hospital, 22101 Moross Rd, Detroit, MI 48236, USA. Email: Mohamad.ayas@ascension.org

doi: https://doi.org/10.14740/gr1336 bowel mesentery (ascending colon: $24 \%$ ) and may extend into the retroperitoneum (14.5\%) [2]. Due to its low incidence rate, they are considered the rarest variety between abdominal cysts. Exact etiology is still unknown, but the most acceptable theory is thought to be a benign proliferation of ectopic lymphatics in the mesentery that lack communication with the remainder of the lymphatic system [1]. Mesenteric cysts are often asymptomatic, however, can present with a variety of nonspecific symptoms or acute abdomen, depending on the tumor size and location [1]. We present a case of an abnormally located mesenteric cyst that was initially thought to be a biloma or possible pancreatic pseudocyst.

\section{Case Report}

A 35-year-old woman with a past medical history of chronic cholelithiasis presented to the hospital with complaints of progressive epigastric/right upper quadrant (RUQ) abdominal pain and nausea for the past few months. Patient had denied any weight loss, loss of appetite, history of alcoholism or pancreatitis and any history of abdominal trauma or surgeries. Patient denied any fevers, chills, vomiting, shortness of breath, chest pain, dysuria, diarrhea, constipation or abdominal distention. Vital signs were stable. Physical exam showed a soft, nondistended abdomen with mild RUQ tenderness, a negative Murphy sign, no rebound tenderness, guarding or hepatosplenomegaly. Laboratory results were remarkable for an aspartate transaminase (AST) of $142 \mathrm{IU} / \mathrm{L}$, an alanine transaminase (ALT) of $278 \mathrm{U} / \mathrm{L}$, alkaline phosphatase (ALP) of $182 \mathrm{IU} / \mathrm{L}$ and a lipase level within normal limits. Computed tomography (CT) scan showed a large cystic mass (Fig. 1) measuring 10.3 $\times 9.8 \times 11.3 \mathrm{~cm}$ in the RUQ/epigastric area with mild intrahepatic biliary ductal dilation. Due to the abnormally located cystic mass, the gastroenterologist was consulted for possible endoscopic intervention. The patient subsequently underwent endoscopic ultrasonography (EUS) for possible drainage of the cyst using an axios stent, instead, a septated cystic structure was seen (Fig. 2). Fine-needle aspiration (FNA) was performed, and $60 \mathrm{~mL}$ of greenish-dark brown fluid was aspirated. The location and color of the fluid aspirated suggested a possible biloma. Fluid was sent to the laboratory for analysis, however, did not reveal the presence of bilirubin, bile salts, carcinoembryonic antigen, or lipase. The general surgeons were consulted and the patient then underwent an exploratory laparotomy revealing a large cyst in the RUQ that was found to be anterior to the duodenum impinging on the gallbladder, cystic duct and on the common bile duct. The cyst had a smooth con- 


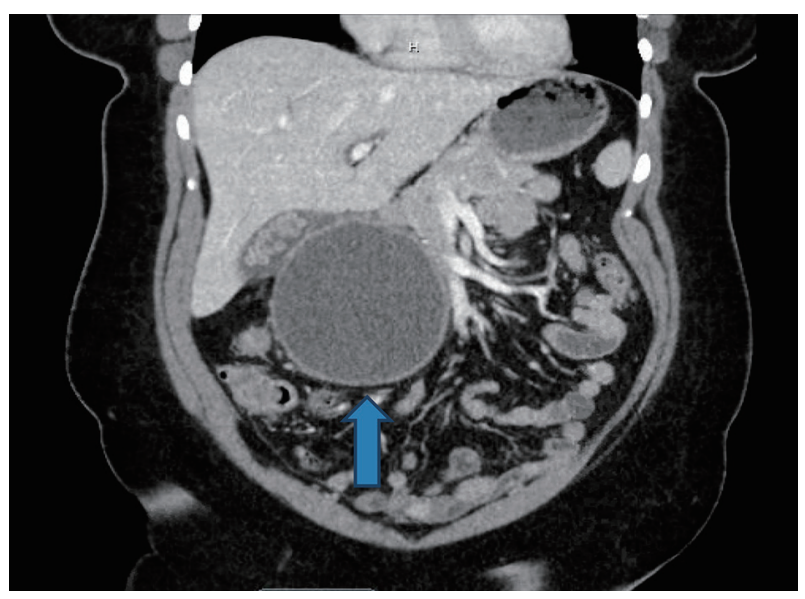

Figure 1. Abdominal computed tomography (CT) scan showing a 10.3 $\times 9.8 \times 11.3 \mathrm{~cm}$ right upper quadrant (RUQ) cystic mass (blue arrow).

sistency without evidence of neoplastic or metastatic disease. The pseudo-capsule of the cyst was taken down the lesser sac, and the cyst was dissected off the root of the mesocolon. The dissection was continued posteriorly, and it was released off of the duodenum. It was then noted that the cyst was impinging onto the porta hepatis as well as the cystic duct, and the gallbladder was found to be adherent to the cyst. At that point, the possibility of a biloma could not be confidently ruled out, and intraoperative cholangiogram was done; however, there was no communication between the cystic mass and biliary tree (Fig. 3). The mass was a 392-g smooth-walled cyst measuring $11.2 \times 8.3 \times 7.5 \mathrm{~cm}$. The outer surface had focal adhesions, the cyst was filled with red soft friable material along with redtinged watery fluid, and the cyst wall was uniform in thickness averaging $0.4 \mathrm{~cm}$. There was no evidence of any bilious or chylous material. The cyst was removed, and concurrent cholecystectomy was performed and a total of two specimens were sent to the laboratory for analysis, the first being the cyst in its entirety, and the second being the gallbladder after resection. Differential diagnosis of this cystic mass included choledochal cyst, pancreatic pseudocyst, echinococcal cyst, cystic mesenteric panniculitis, etc. Pathologic analysis of the cyst was con-

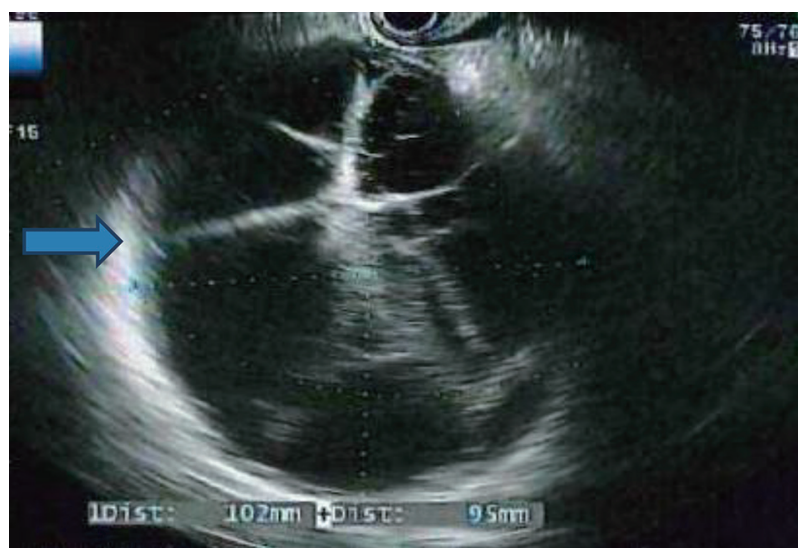

Figure 2. Endoscopic ultrasonography (EUS) revealing a large septated mass (blue arrow).

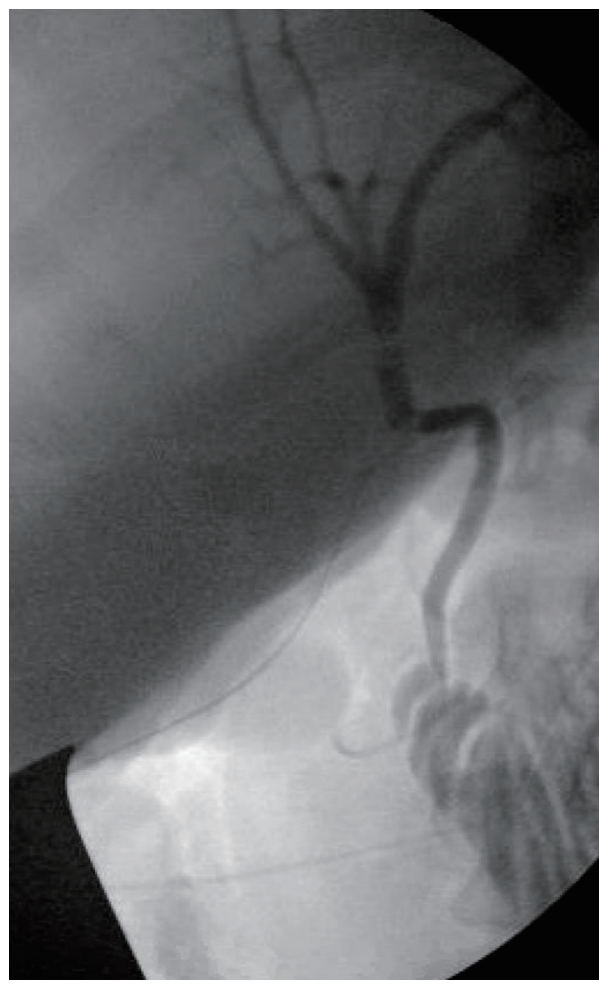

Figure 3. Intraoperative cholangiogram showing lack of dye extravasation between the mass and biliary tree.

sistent with a fibrous wall and prominent lymphoid aggregates, focal scattered foamy histiocytes with a central cavity, filled with blood-tinged fluid (Figs. 4-6). Overall, the features were that of a benign cyst consistent with a mesenteric cyst. After the surgery, the patient was hemodynamically stable, tolerat-

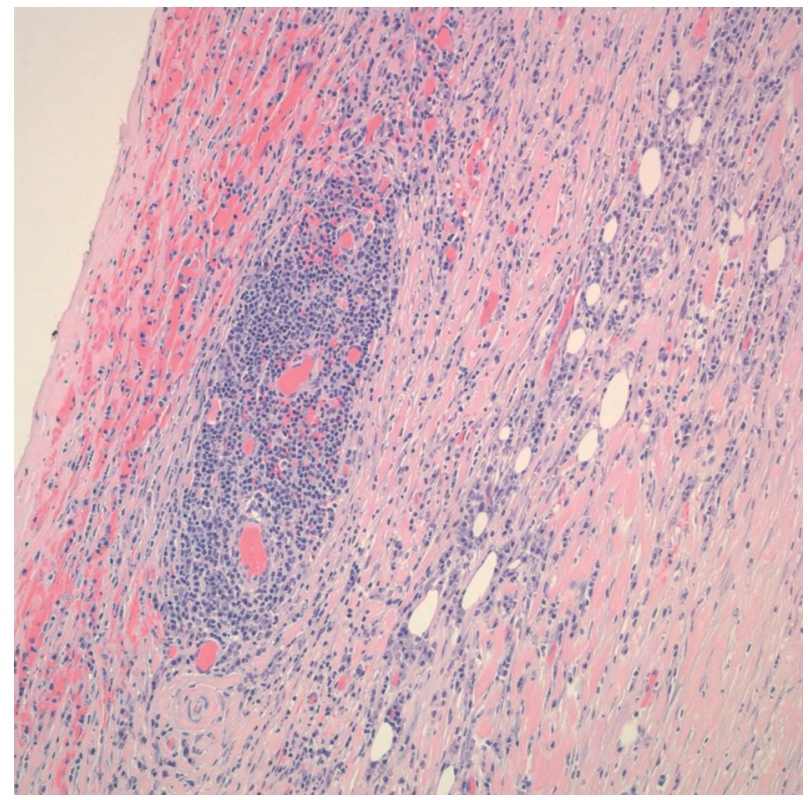

Figure 4. Prominent lymphoid aggregate surrounding small vessels and capillaries within the cyst wall (mid-power views, $\times 20$ ). 


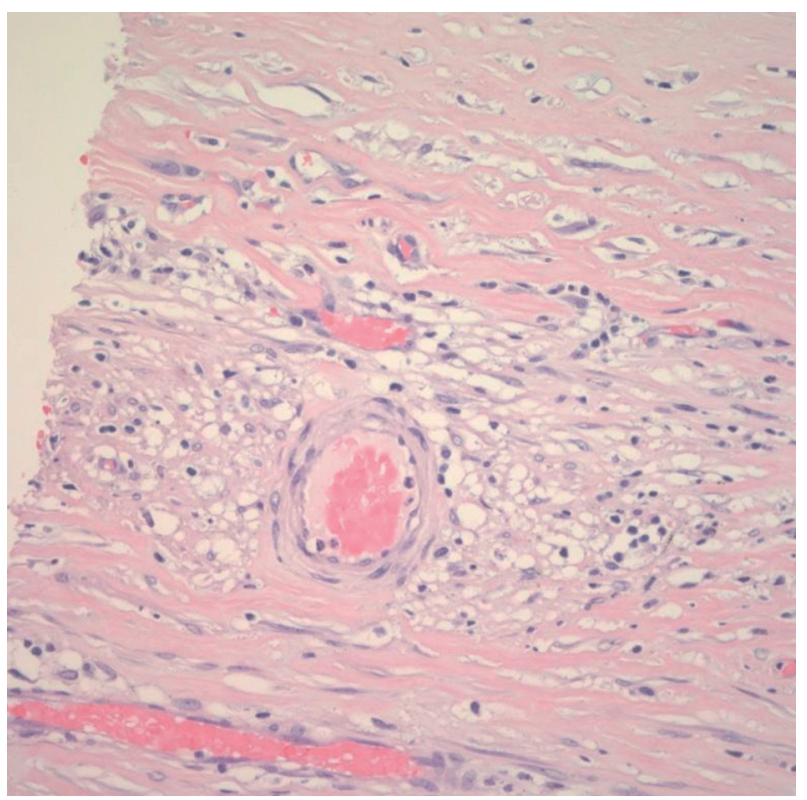

Figure 5. Foamy histocytes surrounding blood vessels within the cyst wall (high power, $\times 40$ ).

ing her diet and was discharged home.

\section{Discussion}

Mesenteric cysts are extremely rare intra-abdominal benign tumors, with approximately 1,000 cases reported in the literature [3]. It was first described by Italian anatomist Benevenni in 1507 while performing an autopsy on an 8-year-old boy [1] Mesenteric cysts can occur at any age, most commonly found in the fourth decade [4], and one-third of cases were found in children younger than 15 years of age [1]. Mesenteric cysts are more common in Caucasians and have a slight female predominance [4]; however, it was seen to have a male predominance $(62.5 \%)$ in the pediatrics age group [5].

Diagnostic modalities vary, although ultrasonography (USG) and CT scan of the abdomen can detect the location, size of the lesion, septation, debris, fluid levels and the thickness of the wall [4], magnetic resonance imaging (MRI) was found to be more specific and is the most accurate investigation. Therefore, MRI is the modality of choice to accurately define the relationship between the mass and its surrounding structures [6].

Treatment of choice for mesenteric cysts is complete surgical resection [1], laparoscopically or via laparotomy. The first successful surgery for a cystic mass in the mesentery was in 1880 by Tillaux, since then, resection has been the mainstay of treatment $[1,7]$. Mackenzie et al in 1993 reported the first laparoscopic excision of a mesenteric cyst [8]. If resection is not possible due to the size or its deep location within the root of the mesentery, partial excision with marsupialization of the cyst into the abdominal peritoneal cavity is also an option [9]. Deroofing and aspiration are not recommended as they are associated with high recurrence and infection rate [3,9]. In our case, we deferred aspiration, as it is very important to take a complete

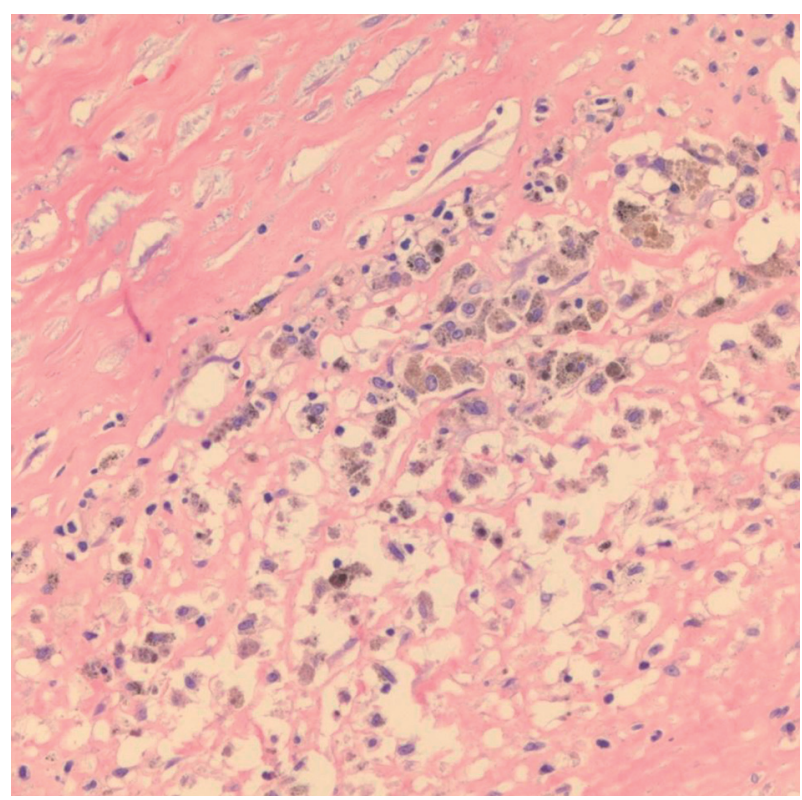

Figure 6. Pigmented and foamy macrophages near the cyst lining (high power, $\times 40$ ).

history, for not every abdominal cyst should be drained.

Mesenteric cysts are the rarest variety of abdominal cysts and due to its low incidence rate, they are usually lower on the differential diagnosis of an abdominal cystic mass. Early diagnosis and treatment is necessary, as malignant transformation is rare but has been described in up to $3 \%$ of cases [8]. Abdominal cysts have a wide spectrum of differential diagnoses, including but not limited to pancreatic pseudocysts, hemangiomas, peritoneal inclusion cysts, cystic mesenteric panniculitis (sclerosing mesenteritis), bilomas, hydatid cysts, cystic teratomas, urogenital cysts [10] and mesenteric cyst being the rarest of them [1]. Mesenteric cysts have a very low recurrence rate when surgically excised (0-13.6\%) and patients have an excellent prognosis [7]. However, further recommendations for guideline-directed therapy are needed for optimal management of mesenteric cysts.

\section{Conclusion}

Mesenteric cysts are benign cysts that are difficult to diagnose due to its rarity, and it is important for it to be differentiated from other abdominal cysts, as management of this entity is treated with surgical excision rather than drainage, as management of this entity is treated with surgical excision rather than drainage. Unfortunately, many abdominal cysts are immediately thought to be pancreatic pseudocysts as they are the most common of the abdominal cysts and are readily drained. That is why one must keep in mind the patient's history and risk factors prior to draining any abdominal cyst.

\section{Acknowledgments}

We would personally like to thank Dr. Juliann Mendes from 
Ascension St. John Hospital, Department of Pathology in Detroit, MI for her great and appreciated contribution and help with providing the pathologic slides with their descriptions.

\section{Financial Disclosure}

There is nothing to declare, and no there are no financial interests or benefits.

\section{Conflict of Interest}

There is no conflict of interest.

\section{Informed Consent}

Informed consent was granted by the patient prior to writing this manuscript.

\section{Author Contributions}

Mohamad Fekredeen Ayas: literature review; preparation, writing and editing of entire manuscript. Saif Affas: literature review; writing of manuscript. Gilles Jadd Hoilat: literature review; writing of manuscript. Ihab Kassab: literature review; assisted with manuscript editing. Mohammed Barawi: corresponding attending and senior author.

\section{Data Availability}

Information was collected from a PubMed search using the keyword "Mesenteric cyst". All other data were collected from the eCare system of Ascension St. John Hospital.

\section{References}

1. Pithawa AK, Bansal AS, Kochar SP. Mesenteric cyst: A rare intra-abdominal tumour. Med J Armed Forces India. 2014;70(1):79-82.

2. Lee DL, Madhuvrata P, Reed MW, Balasubramanian SP. Chylous mesenteric cyst: A diagnostic dilemma. Asian J Surg. 2016;39(3):182-186.

3. Al-Mulhim AA. Laparoscopic excision of a mesenteric cyst during pregnancy. JSLS. 2003;7(1):77-81.

4. Aguirre SV, Mercedes Almagro M, Romero CA, Romero SS, Molina GA, Buenano RA. Giant mesenteric cyst from the small bowel mesentery in a young adult patient. J Surg Case Rep. 2019;2019(1):rjz002.

5. Tamene A, Desta M, Tebeje H, Getie Y, Berhane H. Giant mesenteric cyst and right sided syndrome in a 15-year-old boy. European J Pediatr Surg Rep. 2019;7(1):e5-e7.

6. Guraya SY, Salman S, Almaramhy HH. Giant mesenteric cyst. Clin Pract. 2011;1(4):e108.

7. Bang GA, Tolefac P, Fola O, Biyouma M, Bisay U, Guifo ML, Essomba A. Giant sixteen kilogram lymphangioma mesenteric cyst: An unusual presentation of a rare benign tumour. Int J Surg Case Rep. 2019;59:94-96.

8. Kumar A, Jakhmola CK, Arora NC, Chauhan SS. Mesenteric cyst in sigmoid mesocolon - A rare location and its laparoscopic excision. Med J Armed Forces India. 2015;71(Suppl 2):S425-428.

9. Al-Harfoushi R, Stevenson L, Binnie N. Mesenteric cyst: drained and marsupialised laparoscopically avoiding enterectomy. BMJ Case Rep. 2012;2012.

10. Paramythiotis D, Bangeas P, Karakatsanis A, Iliadis A, Karayannopoulou G, Michalopoulos A. Ideal treatment strategy for chylous mesenteric cyst: a case report. J Med Case Rep. 2018;12(1):317. 